\title{
Sub-nanometer Resolution Chemi-STEM EDS Mapping of Superlattice Intrinsic Stacking Faults in Co-based Superalloys
}

\author{
Michael S. Titus ${ }^{1}$, Akane Suzuki ${ }^{2}$, Michael J. Mills ${ }^{3}$, and Tresa M. Pollock ${ }^{1}$ \\ ${ }^{1}$ University of California, Santa Barbara, Materials Department, Santa Barbara, CA, USA \\ ${ }^{2}$ GE Global Research Center, Schenectady, NY, USA \\ ${ }^{3 .}$ The Ohio State University, Department of Materials Science and Engineering, Columbus, OH, USA
}

With the recent discovery of a ternary $\gamma^{\prime}-\mathrm{Co}_{3}(\mathrm{~A} 1, \mathrm{~W})$ phase of $\mathrm{L}_{2}$ crystal structure, new precipitation strengthened Co-base alloys have been investigated for potential use as gas turbine blades for energy and aero applications [1]. A prevalent deformation mode for turbine blades includes time-dependent plastic deformation, known as creep, which is caused by the centrifugal forces exerted on the blades from the rotating rotor. TEM analysis of interrupted creep specimens of various single crystal Co-based superalloys reveal the presence of numerous superlattice intrinsic stacking faults (SISFs) in the $\gamma^{\prime}$ phase that are remnants of a dislocation shearing events. HRSTEM analysis confirms the nature of the SISFs, as well as compositional variation across the SISFs that must be included in creep deformation models, as shown in Figures 1 and 2.

Early research showed that Co-based superalloys possess a higher melting temperature and improved single crystal solidification characteristics over Ni-based alloys [2,3], which have seen decades of use in advanced gas turbine engines. Co-based alloys, like Ni-based alloys, possess a $\gamma-\gamma$ ' microstructure where relatively undeformable $\gamma^{\prime}\left(\mathrm{L1}_{2}\right)$ precipitates are surrounded by a soft and ductile $\gamma$ (A1) matrix. Mechanical tests have shown that single crystal Co-based alloys are comparable to Ni-based alloys in yield strength and creep resistance at elevated temperatures [4,5]. However, the shearing mechanisms prevalent during creep in Co-based superalloys differ from Ni-based superalloys above $850^{\circ} \mathrm{C}$.

Figure 1a shows a representative micrograph of a crept specimen containing numerous SISFs. When the SISFs are viewed edge-on, parallel to the [011] direction, HAADF HRSTEM images reveal the presence of local HCP stacking consistent with an intrinsic stacking fault (Figure 1c). Due to the ordering of the $\gamma$ ' phase, the four $\{111\}$ atomic planes contained in the SISF are locally D0 ${ }_{19}$ structure (ordered HCP), which is the identical crystal structure of the $\mathrm{Co}_{3} \mathrm{~W}$ phase present in the $\mathrm{Co}-\mathrm{W}$ binary system.

EDS STEM mapping using a FEI Titan $3^{\mathrm{TM}}$ equipped with a Super-X lens and Chemi-STEM system (4x windowless SDDs with a total area of $150 \mathrm{~mm}^{2}$ ) reveals the presence of slight changes in composition near the SISFs (Figure 2). Integrated line scans taken across the SISF clearly show the enhancement of $\mathrm{W}$ and $\mathrm{Ta}$, and the depletion of $\mathrm{Co}$ and $\mathrm{Al}$ in the vicinity of the fault. Near the fault, the composition shifts from the $\gamma^{\prime}$ phase to the nearby $\mathrm{Co}_{3}(\mathrm{~W}, \mathrm{Ta})-\mathrm{D}_{19}$ phase. Integrated line scans (Figure 2) and $\mathrm{z}$-contrast HAADF images (Figure $1 \mathrm{~b}$ ) show that the change in composition is highly localized to the four atomic planes forming the SISF.

While the exact mechanism of shearing that produces SISFs in Co-based alloys is currently unknown, models for shearing in Ni-based alloys have been developed. One mechanism includes a diffusionmediated reordering process that converts an antiphase boundary to a SISF in the $\gamma^{\prime}$ phase [6]. This process would alter the local chemistry of the SISF in Ni-based alloys. Our analysis of the SISFs in various Co-based alloys suggests that diffusion is occurring along the faults, which must be accounted 
for in any creep deformation models. New alloying strategies for increasing creep strength and progress towards quantitative EDS mapping will be presented.

\section{References:}

[1] J Sato, T Omori, K Oikawa, I Ohnuma, R Kainuma, K Ishida, Science 312 (2006), p. 90.

[2] TM Pollock, J Dibbern, M Tsunekane, J Zhu, A Suzuki, JOM 62 (2010), p. 58.

[3] M Tsunekane, A Suzuki, TM Pollock, Intermet 19 (2011), p. 636.

[4] MS Titus, A Suzuki, TM Pollock, Acta Mater 56 (2012), p. 1288.

[5] MS Titus et al, Superalloys 2012 (2012) p. 823.

[6] VA Voronstov, L Kovarik, MJ Mills, CMF Rae, Acta Mater 60 (2012), p. 4866.

[7] The authors acknowledge funding from NSF DMREF Grant DMR 1233704 and General Electric.
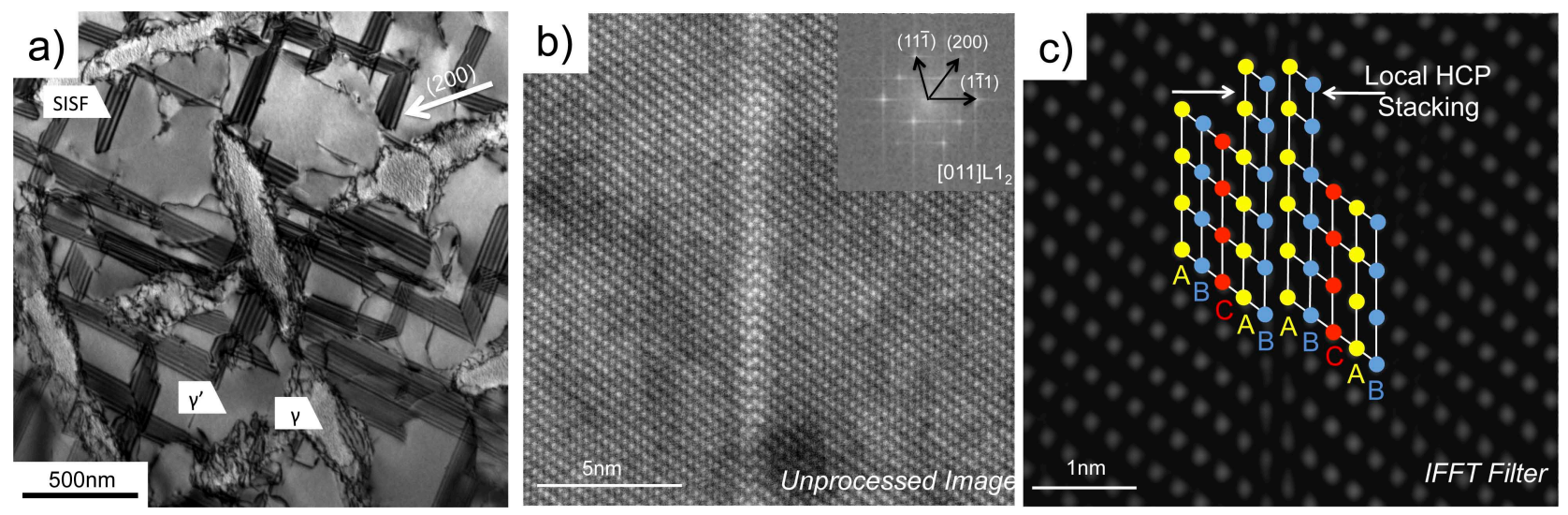

Figure 1. TEM bright field image (a) of a Co-8.8Al-9.8W-2Ta (at.\%) single crystal alloy crept to $2 \%$ at $900^{\circ} \mathrm{C}$ and $345 \mathrm{MPa}$. Numerous SISFs are observed within individual $\gamma$ ' precipitates. HAADF STEM image (b) of a SISF viewed edge-on, parallel to the [011] direction. IFFT filtered image shown (c) of region from (b). Local HCP stacking is observed along the SISF.

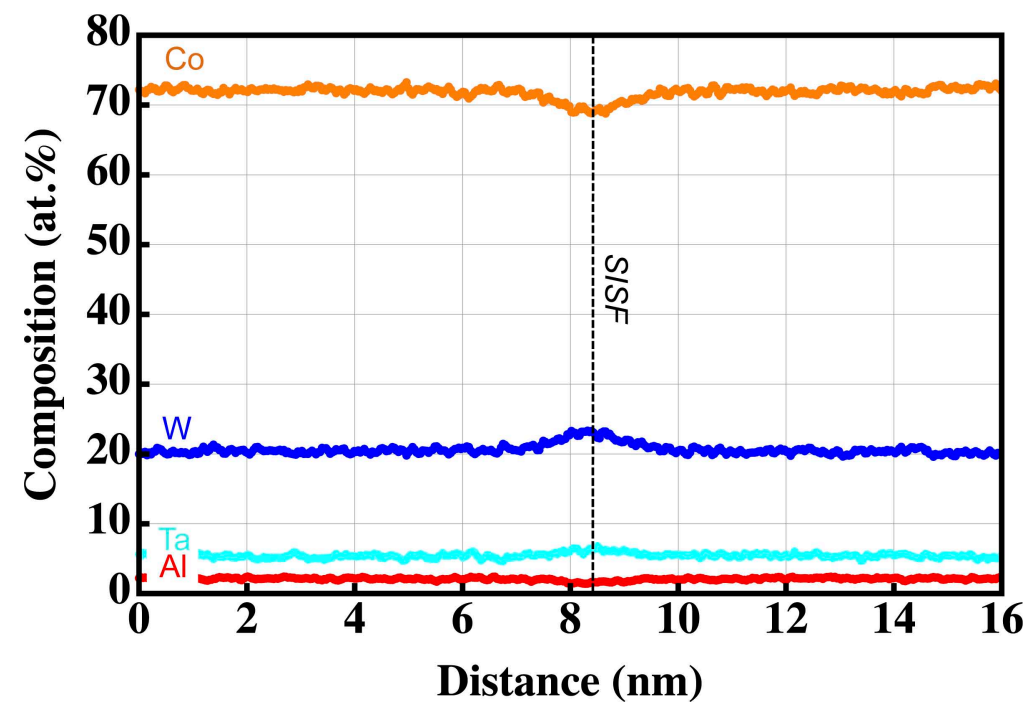

Figure 2. Integrated line scan over the SISF in Fig. 1(b) showing local composition changes within $2.5 \mathrm{nms}$ of the fault. Compositional analysis was carried out using standard $\mathrm{k}$ factors within the Bruker ESPIRIT software package. 